\title{
EXPRESSION OF PD-L1 AND IMMUNE CELLS IN GASTROINTESTINAL STROMAL TUMOURS
}

\author{
Alexandra ROSULESCU1,2凶, Mariana COSTACHE ${ }^{1,3}$, Adrian DUMITRU ${ }^{1,3}$, Maria SAJIN ${ }^{1,3}$ \\ ${ }^{1}$ Department of Pathology, Faculty of Medicine, "Carol Davila" University of Medicine and Pharmacy, \\ Bucharest, Romania \\ ${ }^{2}$ Department of Pathology, Sanador Hospital, Bucharest, Romania \\ ${ }^{3}$ Department of Pathology, University Emergency Hospital, Bucharest, Romania
}

Received 29 July 2021, Accepted 27 Aug 2021

https://doi.org/10.31688/ABMU.2021.56.3.01

\section{Abstract}

Introduction. Tumour microenvironment and the expression of check-point molecules on tumour cells was proven to have prognostic and therapeutic implications in various types of cancer. Evaluation of immune infiltrate and Programmed Death-1 (PD-L1) expression in gastrointestinal stromal tumours (GISTs) could bring valuable information for development of targeted immune therapy for a subset of cases.

The objective of this study was to determine the PD-L1 expression and to analyse the intratumoural inflammatory infiltrate in different categories of GISTs.

Material and methods. Twenty four cases of immunohistochemically confirmed GISTs were microscopically reviewed and tested for PD-L1, CD3 and CD20. Their expression was quantified and statistical analysis was performed to search for differences between subgroups.

Results. The expression of PD-L1 on tumour cells was identified in fourteen cases, with variable intensity and extent, ten cases being negative. The immune infiltrate was invariably present, lymphocytic predominant, with intertumoural and intratumoural

\section{Résumé}

L'expression de PD-L1 et les cellules immunitaires dans les tumeurs stromales gastro-intestinales

Introduction. Le micro-environnement tumoral et l'expression des molécules des check-point sur les cellules tumorales ont était prouvé d'apporter des effets sur le plan thérapeutique et pronostic dans certains types de cancer. L'évaluation de cellules immunitaires et l'expression de PD-L1 dans les tumeurs stromales gastro-intestinales (GIST) pourrait se rendre très utile dans la prise en charge d'une immunothérapie ciblé pour certains patients.

L'objectif de cette étude était de déterminer l'expression de PD-L1 et d'analyser l'infiltrat inflammatoire intratumoral dans différentes catégories de GIST.

Matériel et méthodes. Vingt-quatre cas de GIST confirmés par immunohistochimie ont été examinés au microscope et testés pour PD-L1, CD3 et CD20. Leur expression a été quantifiée et une analyse statistique a été effectuée pour rechercher des différences entre les sous-groupes. 
quantitative differences, from sparse to abundant and with focal aggregation around blood vessels, fibrous septa, in the periphery of the tumour and under ulcerated areas. $T$ cells were the dominant lymphocytic subtype. Causality connections could not be determined between PD-L1 expression, the pattern of inflammation and other clinical and pathological variables, limitation determined in part by the reduced extent of the study.

Conclusions. GISTs express PD-L1 in a significant percentage of cases and have a great variability in the number and distribution of intratumoural immune cells, aspects that can be further exploited for therapeutic purposes.

Keywords: gastrointestinal stromal tumour, checkpoint molecules, PD-L1, immune cells, immunotherapy, lymphocytes

\section{List of abbreviations:}

GIST - gastrointestinal stromal tumour

PD-1 - Programmed Death-1

PD-L1- Programmed Death-Ligand1

CD3 - cluster of differentiation 3

CD20 - cluster of differentiation 20

$H \& E$ - hematoxylin and eosin

HPF - high power field

TKI - tyrosin-kinase inhibitors

\section{INTRODUCTION}

Gastrointestinal stromal tumours (GISTs) are mesenchymal tumours originating from interstitial cells of Cajal, determined by function mutations in kit receptor tyrosine kinase, in more than $75 \%$ of cases $^{1,2}$. After their pathogenesis was discovered, targeted molecular therapy with tyrosine kinase inhibitors, such as Imatinib, Sunitinib and Regorafenib, became an effective therapy, improving the overall survival of patients diagnosed with high risk GISTs. Nonetheless, a subset of cases are refractory to treatment, and novel therapeutic strategies are being under investigation ${ }^{3,4}$.

Malignant tumours are highly mutagenic and adaptable. In the evolution of cancer, subclones emerge and develop, inducing intratumoural modifications, that lead to treatment resistance. In this context, therapy directed against tumour cells alone is not able to keep up with the perpetual changes. As immune response is adaptable, being able to detect new mutations and fight against cells harbouring them, immune therapy could be a solution in cases refractory to conventional treatment ${ }^{5,6}$.
Resultats. L'expression de PD-L1 sur les cellules tumorales a été identifiée dans quatorze cas, avec une intensité et une étendue variables, dix cas étant négatifs. L'infiltrat immunitaire était invariablement présent, lymphocytaire à prédominance, avec des différences quantitatives intertumorales et intratumorales, de clairsemé à abondant et avec agrégation focale autour des vaisseaux sanguins, fibrose septa, en périphérie de la tumeur et sous les zones ulcérées. Les lymphocytes $T$ étaient le sous-type lymphocytaire dominant. Les liens de causalité n'ont pas pu être déterminés entre l'expression PD-L1, le modèle de l'inflammation et d'autres variables cliniques et pathologiques, limitation déterminée en partie par l'ampleur réduite de l'étude.

Conclusions. Les GIST expriment PD-L1 dans un pourcentage significatif de cas et ont une grande variabilité dans le nombre et la distribution des cellules immunitaires intratumorales, aspects qui peuvent être davantage exploités à des fins thérapeutiques.

Mots-clés: tumeur stromale gastro-intestinale, molécules des check-point, PD-L1, cellules immunitaires, immunothérapie, lymphocytes

Recent advances in the field of immunotherapy are regarding immune checkpoints, molecules that play an important role in the activation or inhibition of T cells activity. Such a checkpoint molecule is Programmed Death-1 (PD-1), a surface receptor expressed on activated lymphocytes, acting as a down-regulator of the immune system. His ligand, Programmed Death-Ligand1 (PD-L1) expressed on tumour cells and on antigen-presenting cells from tumour microenvironment, inhibits $\mathrm{T}$ cells activity, PD-1/PD-L1 pathway playing an important role in immune tolerance and thus tumour progression ${ }^{7 \cdot 10}$.

PD-1 blockade was tested and approved for the first time in the case of metastasizing malignant melanoma. This strategy was also efficient in non-small cell lung carcinomas, renal cancers, hypermutated gastrointestinal cancers and others. Immunohistochemical analysis of PD-L1 expression on tumour cells proved to be useful in selecting the patients for such therapies ${ }^{11-15}$. In soft tissue sarcoma, the significance of PD-L1 expression for prognosis and as a therapeutic approach is unknown at the present time ${ }^{16}$.

With the development of these new immune therapeutic lines and in the context of resistance to 
classical treatment, GISTs could also be considered for evaluation of checkpoint blockade possibilities and for defining a relationship with the microenvironment, especially with the antitumoural immune response $\mathrm{e}^{17,18}$.

The significance of immune infiltrate in GISTs has not been extensively studied and its prognostic role is not fully understood. A limited number of studies show that the immune population is predominantly represented by macrophages and T-cells. B-lymphocytes and natural killer cells are sparse, but have a higher density in metastases. Additionally, Imatinib therapy was shown to influence the immune response by activating the cytotoxic T-cells, suggesting a possible significant contribution of the immune system in the treatment of GISTs ${ }^{19-21}$.

THE AIM OF THIS STUDY was to evaluate the expression of PD-L1 in GISTs, to examine the intratumoural immune infiltrate, and to find possible correlations with other clinical-pathological variables and prognosis.

\section{Material AND MEthods}

Twenty four GISTs from twenty four patients were included in this retrospective study. All cases were registered in the Pathology Department of the University Emergency Hospital Bucharest, Romania, after being diagnosed in the same service between $1^{\text {st }}$ of January 2015 and $1^{\text {st }}$ of July 2018. An informed consent was signed by all involved patients, allowing the use of their tissues for scientific studies.

The clinical data and pathological features were analysed using clinical records and pathology reports. Useful clinical data consisted of age of the patient, sex, tumour location, symptoms, medical history. Pathology reports mentioned tumour size, cellular type, mitotic rate, presence or absence of ulceration, prognostic group and tumour stage according to World Health Organization (WHO) Classification of Digestive System Tumours 2019 (5 th $^{\text {th }}$ edition)/ American Joint Committee on Cancer 2017 ( $8^{\text {th }}$ edition ${ }^{22,23}$. Original histologic slides were reviewed from the archives for observing certain details not mentioned in the official report, as the quantity and type of the inflammatory infiltrate present in the tumour. The study included exclusively immunohistochemically-confirmed GISTs.

To assess the expression of PD-L1 in selected GISTs, we performed immunohistochemical testing. Formalin-fixed and paraffin-embedded specimens were sectioned at $4 \mu \mathrm{m}$ thickness and mounted on glass slides. The PD-L1 antibody (clone CAL10) was obtained from Biocare. Positive expression was defined as membranous and/or cytoplasmic staining of
$>1 \%$ of the tumour cells visualized. A quantification system for PD-L1 positivity was adopted: 1 if $1-10 \%$ of tumour cells are positive, 2 if $11-50 \%$ of tumour cells stain positive and 3 if more than $50 \%$ of cells qualify as positive ${ }^{24}$.

Immune infiltrate was initially evaluated on hematoxylin and eosin (H\&E) stained slides. The quantity of immune infiltrate was scored as 1,2 and 3 (minimum, medium and abundant). The infiltrate designated as minimum can be translated as only sparse, isolated immune cells, no more than 20 cells/ high power field (HPF), moderate means a higher density of immune cells (up to 500 cells/HPF) and/ or sparse aggregates of immune cells and abundant infiltrate is the equivalent of contiguous areas were the immune cells prevail (more than 500 cells/HPF). Further on, CD3 and CD20 expression of immune cells was evaluated by immunohistochemistry, in the attempt to characterize the immune response in selected tumours.

Statistical analysis was performed using Microsoft Excel program. The Chi-square test was used for comparison between categorical variables. Spearman's rank correlation coefficient was calculated to define the relationship between the prognostic group, PD-L1 expression, and immune infiltrate. A p level less than 0.05 was accepted as statistically significant.

\section{Results}

The sex distribution of the 24 patients diagnosed with GISTs showed 11 males and 13 females, aged between 29 and 76 years old, with a median age of 63.5 years. Tumours had gastric location in 16 cases, small intestine in 6 cases and 2 were extragastrointestinal: one in the omentum and one in the peritoneum. Tumour dimensions varied between $1 \mathrm{~cm}$ and $19 \mathrm{~cm}$ in greatest diameter. Corroborating location, size and mitotic activity, evaluated on $50 \mathrm{HPF}$, the cases were included in prognostic groups, according to Armed Forces Institute of Pathology criteria ${ }^{22}$. Accordingly, one case fulfilled the criteria for the $1^{\text {st }}$ group, six cases for the $2^{\text {nd }}$ group, eight were included in group 3a, one in 3b, one in 4, two in 5, two in 6a and three in $6 \mathrm{~b}$ prognostic groups. As for the histologic type, 19 GISTs were composed of spindle cells, two were epithelioid and three were of mixed subtype. Pleomorphism was absent in 3 cases, subtle in 15 cases and moderate in 6 of the analysed cases.

\section{PD-L1 expression in tumour cells}

From the total number of 24 GISTs tested for PD-L1, 14 were positive and 10 were negative. Membranous and/or cytoplasmic pattern of staining was validated as positive if present in more than $1 \%$ 


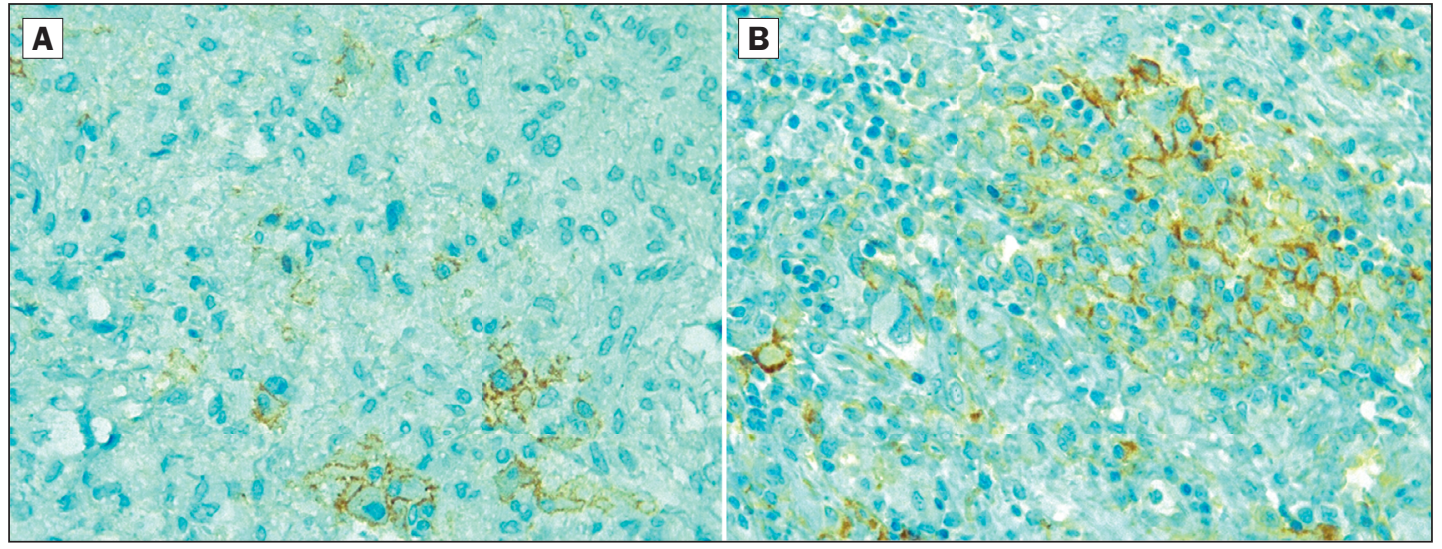

Figure 1. Positive immunohistochemical staining for PD-L1 in GIST (40X). A) Sparse tumour cells with membranous expression; B) Contiguous positive cells.

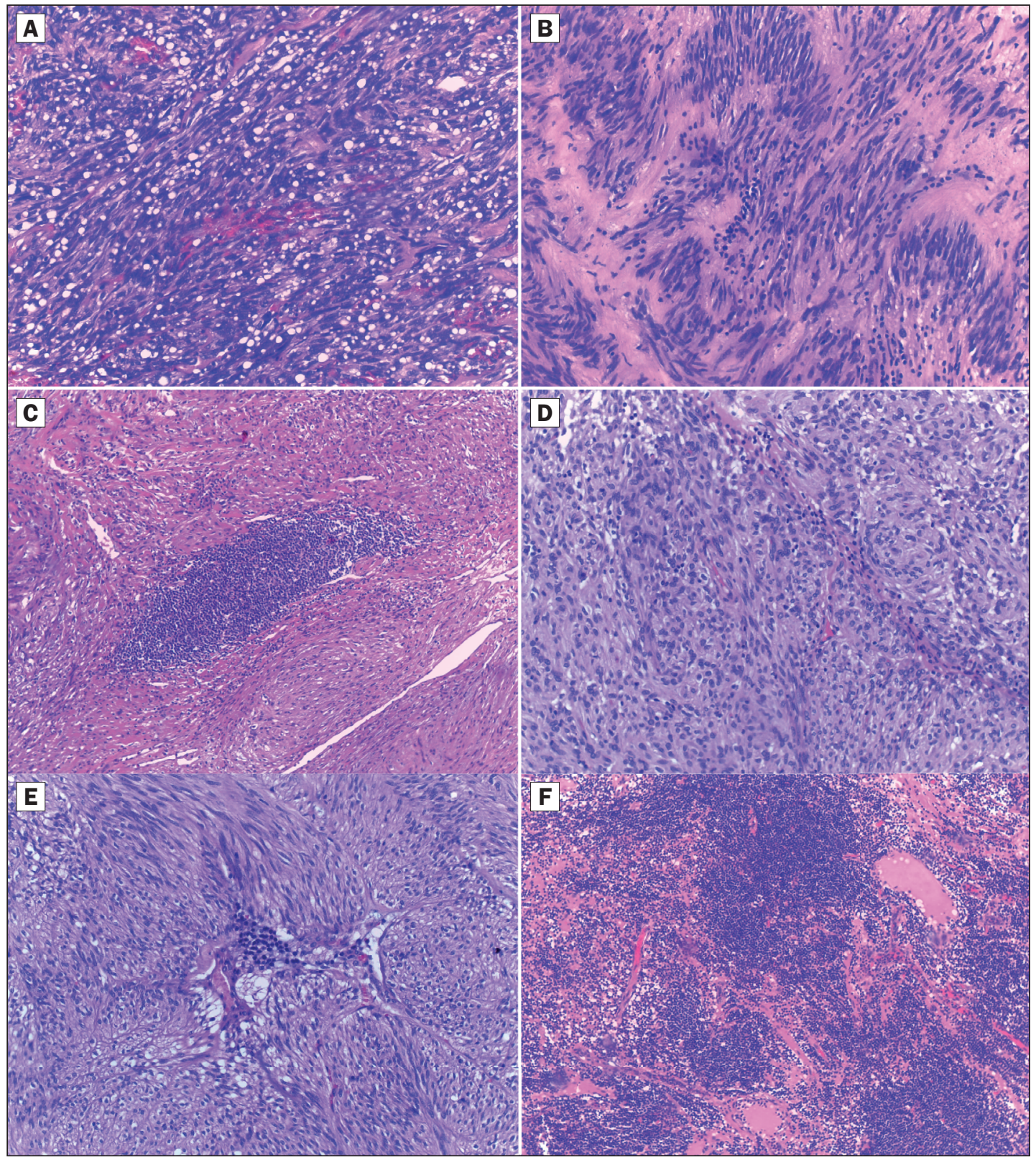

Figure 2. Patterns of inflammation in GIST (H\&E staining). A. Sparse, hardly detectable lymphocytes (40x); B. Diffuse, moderate inflammation (40x); C. Lymphoid aggregates (10x); D. Densification along fibrous septa (40x); E. Perivascular agglomeration (40x); F. Diffuse, abundant lymphocytes infiltration (10x). 


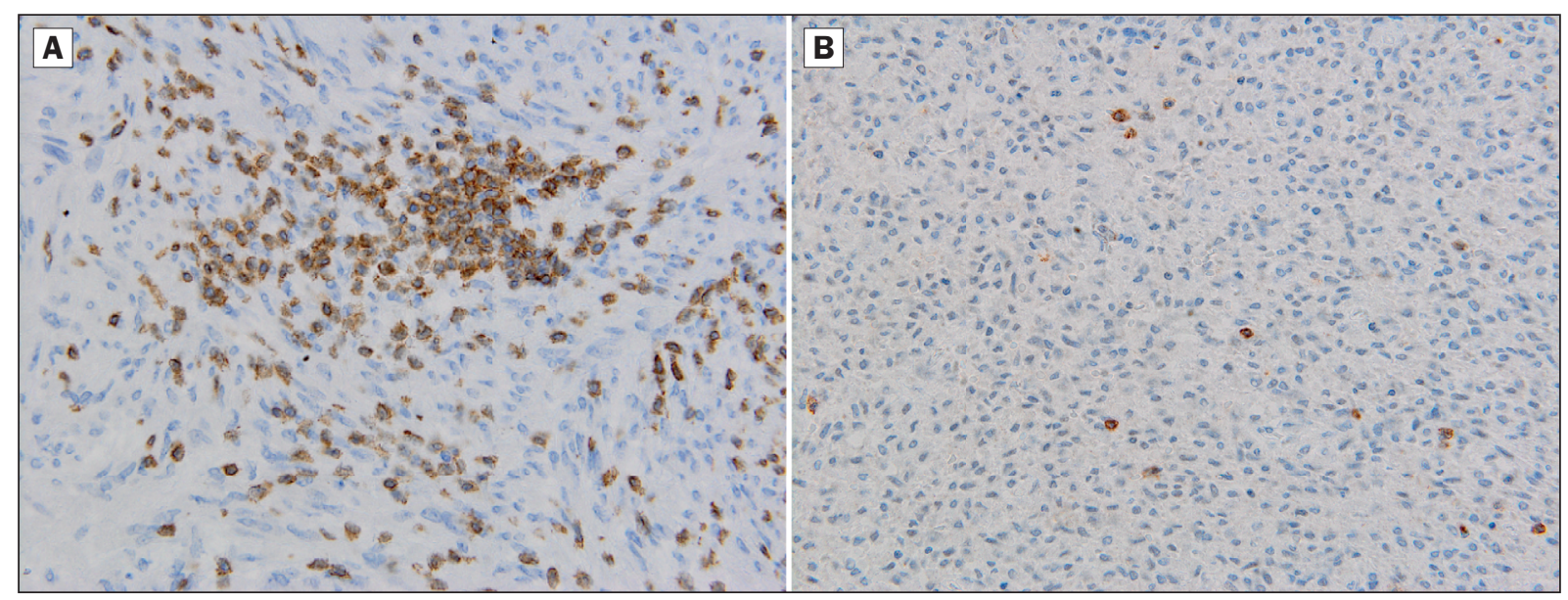

Figure 3. Lymphocyte distribution in GIST - immunohistochemistry images (40X).

A) Dense CD3 + cells. B) Sparse CD20+ cells

Table 1. PD-L1 and immune infiltrate scoring for the analysed cases

\begin{tabular}{cccccccccccccc}
\hline & \multicolumn{3}{c}{ PD-L1 expression } & \multicolumn{3}{c}{$\begin{array}{c}\text { Immune infiltrate } \\
\text { (HEE) }\end{array}$} & \multicolumn{3}{c}{ CD3+ cells } & \multicolumn{3}{c}{ CD20+ cells } \\
\hline Score & 0 & 1 & 2 & 3 & 1 & 2 & 3 & 1 & 2 & 3 & 1 & 2 & 3 \\
\hline Number of cases & 10 & 4 & 8 & 2 & 7 & 13 & 4 & 9 & 15 & 0 & 8 & 16 & 0 \\
\hline
\end{tabular}

of tumour cells. PD-L1 expression was evaluated exclusively on tumour cells. Registered scores for positive cases were $1(n=4), 2(n=8)$ and $3(n=2)$. PD-L1 expression could not be related to cells morphology (i.e. cell type, pleomorphism) or prognostic group (Figure 1).

\section{Inflammatory infiltrate in GISTs}

The immune infiltrate in GISTs showed significant variation, from sparse and focal to diffuse and abundant. Also, the intratumoural distribution of immune cells was variable. A higher density could be observed perivascular, in hemorrhagic areas, accompanying sclerosis and hyalinization, in the periphery of the tumour, creating a peripheral rim and underlying ulcerated areas.

The nature of the inflammatory infiltrate was also variable. Lymphocytes were the dominant component, followed by a limited number of plasma cells, histiocytes, neutrophils and eosinophils. From the quantitative point of view, seven cases were scored with 1,13 with 2 and four with 3 , no tumour showing absolute lack of immune cells. The score reflected the dominant intratumoural aspect (Figure 2).

Immunohistochemical analysis for CD3 and CD20, performed for evaluating the lymphocytic infiltrate, showed both T-cells (CD3+) and B-cells $(\mathrm{CD} 20+)$ in all tumours, with an evident dominance of T-lymphocytes. The distribution was similar to the one described on $\mathrm{H} \& \mathrm{E}$ slides, except that
B-lymphocytes were prevalent in isolated intratumoural lymphoid aggregates (Figure 3).

No correlations were observed between intratumoural immune cells type and quantity and PD-L1 expression or other clinicopathological analysed aspects (Table 1).

\section{Discussion}

GISTs have a wide spectrum of biologic behaviours, from virtually benign to highly aggressive tumours, with the capacity of recurrence and metasta$\mathrm{ses}^{25}$. Armed Forces Institute of Pathology divides the aggressiveness of GISTs in eight classes, from 1 to $6 \mathrm{~b}$, taking into account the size of the tumour, the mitotic index, and tumour location. The treatment depends on risk stratification. Surgical resection is, in most cases, the first line of treatment, followed by adjuvant therapy with tyrosin-kinase inhibitors (TKI) in cases with a high risk of disease progression ( $3 \mathrm{a}$ and above) or when metastases are present. Although Imatinib was proven to increase the survival, most metastatic cases develop resistance. New-generation kinase-inhibitors were developed over time to treat resistant cases, but they proved a large spectrum of side effects. In this context, new lines of treatment must be explored to fight these aggressive, dynamic tumours and one path could be related to the immunologic landscape of the tumour $22,26,27$.

Checkpoint blockade therapy is a form of immunotherapy that acts on the final phase of immune 
response, prolonging it. PD-1 is such a checkpoint molecule, expressed on immune cells and acting as an inhibiting receptor. PD-L1, its ligand, is expressed on tumour cells and when the interaction receptor-ligand takes place, the tumour is safe from the antitumoural immune response $\mathrm{e}^{8,28}$.

Before considering checkpoint blockade therapy in GISTs, PD-L1 expression on tumour cells has to be determined. Our study included 24 cases of GISTs with gastric, small intestine and extragastrointestinal location. We demonstrated that PD-L1 expression is a frequent finding, 14 cases showing PD-L1 positivity of variable intensity and extension, graded as 1 if $1-10 \%$ of analysed cells were positive, 2 if $11-50 \%$ of tumour cells were positive and 3 if more than 50\% of cells were positive. Seifert et al. also proved, in a study on 85 cases, that PD-L1 expression in GIST tumour cells was variable, in most cases low and focal ${ }^{4}$. Sun et al. found that PD-L1 expression was higher in tumours with lesser dimensions and in the epithelioid and mixed morphology compared to spindle cell type $^{29}$. Regarding the significance of PD-L1 positivity, some authors have demonstrated that it represents an independent poor prognostic factor in GIST, while others have concluded that PD-L1 expression was higher in low-risk tumours and in tumours without metastatic relapse ${ }^{30-31}$. We could not identify a relationship between PD-L1 grade and other clinical and pathological aspects of the tumours. As an example, grade 3 was encountered also in gastric and small intestine tumors, small and large tumors, different age groups etc. Considering the limited extent of our study, prognostic assumptions regarding the value of PD-L1 are not possible at the present time.

An issue closely related to PD-L1 expression is the inflammatory infiltrate and immune surveillance. The study of intratumoral lymphocytes in various forms of cancer proved to be associated with a positive effect on prognosis ${ }^{32,33}$. In this research, we analysed the immune infiltrate, invariably present, with emphasis on quantity and distribution. Lymphocytes represented the majority of immune cells accompanied by few plasma cells, histiocytes, neutrophils and eosinophils. The main lymphocytes subtype was represented by T-cells (CD3+). Immune cells were unevenly distributed, from areas with scattered elements to aggregates and zones dominated by the inflammatory infiltrate. We observed a greater density of inflammatory elements around blood vessels, in the periphery of the tumour, in areas of fibrosis and underlying ulcerated surfaces. Lymphoid aggregates showed a predominance of B-cells (CD20+). Our findings are similar to other studies on GIST microenvironment, showing that lymphocytes and histiocytes

are the main intratumoural inflammatory cells and T-cells the dominant lymphocytic subtype ${ }^{20,29}$.

The assessment of the immune response is a very challenging task, being hardly reproducible and impossible to standardize without specialized digital programs. For this reason, our evaluation was more a descriptive one, focusing on inflammation patterns. The evaluation of the dynamic relationship between tumour cells, their microenvironment and immune cells could provide valuable insights in future studies.

\section{Conclusions}

The expression of PD-L1 is frequently detected in GISTs, with no regard to tumour location, prognostic group, or other clinical and pathological variables that characterize the tumour. Its role in prognosis and as a possible therapeutic target is yet unknown.

Inflammatory infiltrate is invariably present in GISTs, with intratumoural and intertumoural variations, presenting under a wide spectrum, from sparse to dense, independently of PD-L1 expression. Both CD3+ and CD20 + intratumoural lymphocytes are present, $\mathrm{CD} 3+$ being the dominant component.

\section{Author Contributions:}

Conceptualization, A.R. and M.C.; methodology, A.R. and A.D.; software, A.R. and A.D.; validation, M.C. and M.S.; formal analysis, A.R. and A.D.; investigation, A.R and A.D.; resources, -; writing-original draft preparation, A.R.; writing-review and editing, A.R., A.D., M.C.; visualization, A.R., A.D., M.C. and M.S.; supervision, M.S.; All the authors have read and agreed with the final version of the article.

\section{Compliance with Ethics Requirements:}

"The authors declare no conflict of interest regarding this article"

"The study was approved by the Ethics Committee of the Emergency University Hospital, Bucharest, Romania (11285/26.02.2021)."

"The authors declare that all the procedures and experiments of this study respect the ethical standards in the Helsinki Declaration of 1975, as revised in 2008(5), as well as the national law. Informed consent was obtained from all the patients included in the study"

"No funding for this study"

\section{Acknowledgements:}

None 


\section{References}

1. Miettinen M, Lasota J. Gastrointestinal stromal tumors. Gastroenterol Clin North Am. 2013; 42(2): 399-415.

2. Corless CL. Gastrointestinal stromal tumors: what do we know now? Modern Pathology. 2014; 27: 1-16.

3. Trent JC, Subramanian MP. Managing GIST in the imatinib era: optimization of adjuvant therapy. Expert Rev Anticancer Therapy. 2014; 14:1445-1459.

4. Seifert AM, Zeng S, Zhang JQ et al. PD-1/PD-L1 blockade enhances $T$ cell activity and antitumor efficacy of imatinib in gastrointestinal stromal tumors. Clin Cancer Res. 2017; 23(2): 454-465.

5. Chen DS, Mellman I. Oncology meets immunology: the cancer-immunity cycle. Immunity. 2013; 39(1):1-10.

6. Schumacher TN, Schreiber RD. Neoantigens in cancer immunotherapy. Science. 2015; 348:69-74.

7. Topalian SL, Drake CG, Pardoll DM. Targeting the PD-1/ B7-H1 (PD-L1) pathway to activate anti-tumor immunity. Curr Opin Immunol. 2012; 24(2):207-212.

8. Tumeh PC, Harview CL, Yearley JH et al. PD-1 blockade induces responses by inhibiting adaptive immune resistance. Nature. 2014; 515:568- 571.

9. Ribas A, Hu-Lieskovan S. What does PD-L1 positive or negative mean? J Exp Med. 2016; 213(13): 2835-2840.

10. Wieder T, Eigentler T, Brenner E, Rocken M. Immune checkpoint blockade therapy. J Allergy Clin Immunol. 2018; 142(5): 1403- 1414.

11. Kleffel S, Posch C, Barthel SR et al. Melanoma cell-intrinsic PD-1 receptor functions promote tumor growth. Cell. 2015; 162:1242-1456.

12. Reck M, Rodriguez-Abreu D, Robinson AG et al. Pembrolizumab versus chemotherapy for PD-L1-positive non-small-cell lung cancer. N Engl J Med. 2016; 375:1823-1833.

13. Kerr KM, Hirsch FR. Programmed Death Ligand-1 immunohistochemistry. Friend or foe? Arch Pathol Lab Med. 2016; 140(4):326-331.

14. Topalian SL, Hodi FS, Brahmer JR, et al. Safety, activity, and immune correlates of anti-PD-1 antibody in cancer. $N$ Engl J Med. 2012; 366:2443-2454.

15. Ostrand-Rosenberg S, Horn LA, Haile ST. The programmed death-1 immune suppressive pathway: barrier to anti-tumor immunity. J Immunol. 2014; 193(8): 3835-3841.

16. D'Angelo SP, Shoushtari AN, Agaram NP, et al. Prevalence of tumor-infiltrating Lymphocytes and PD-Llexpression in the soft tissue sarcoma microenvironment. Hum Pathol. 2015; 46(3):357-365.

17. Rusakiewicz S, Semeraro M, Sarabi M, et al. Immune infiltrates are prognostic factors in localized gastrointestinal stromal tumors. Cancer Res. 2013; 73(12):3499-3510.

18. Cameron S, Haller F, Dudas J, et al. Immune cells in primary gastrointestinal stromal tumors. Eur J Gastroenterol Hepatol. 2008; 20:327-334.

19. Van Dongen M, Savage ND, Jordanova ES, et al. Anti-inflammatory M2 type macrophages characterize metastasized and tyrosine kinase inhibitor-treated gastrointestinal stromal tumors. International Journal of Cancer. 2010; 127: 899-909.

20. Tan Y, Garcia-Buitrago MT, Trent JC, Rosenberg AE. The immune system and gastrointestinal stromal tumor: a wealth of opportunities. Curr Opin Oncol. 2015; 27(4):338-342.

21. Balachandran VP, Cavnar MJ, Zeng S, et al. Imatinib potentiates antitumor $\mathrm{T}$ cell responses in gastrointestinal stromal tumor through the inhibition of Ido. Nat Med. 2011; 17:1094-1100.

22. Dei Tos AP, Hornick JL, Miettinen M. Gastrointestinal stromal tumours. In: WHO Classification of digestive system tumors. 5th ed, International Agency for Research on Cancer, Lyon, 2019, 439-443.

23. DeMatteo RP, Maki RG, Agulnik M, et al. Gastrointestinal stromal tumor. In: Amin MB, Edge S, Greene F et al. AJCC Cancer Staging Manual (8th edition), Springer International Publishing: American Joint Commission on Cancer, Chicago, 2017, 523-529.

24. Roberts JA, Gonzalez RS, Das S, Berlin J, Shi C. Expression of PD-1 and PD-L1 in poorly differentiated neuroendocrine carcinomas of the digestive system: a potential target for anti-PD-1/PD-L1 therapy. Hum Pathol. 2017; 70:49-54.

25. Khoo CY, Chai X, Quek R, Teo MCC, Goh BKP. Systematic review of current prognostication systems for primary gastrointestinal stromal tumors. Eur J Surg Oncol. 2018; 44(4):388-394.

26. Casali PG, Fumagalli E, Gronchi A. Adjuvant therapy of gastrointestinal stromal tumors (GIST). Curr Treat Options Oncol. 2012; 13:277-284.

27. Balachandran VP, DeMatteo RP. GIST tumors: Who should get imatinib and for how long? Adv Surg. 2014; 48(1): 165-183.

28. Taube JM, Klein A, Brahmer JR, et al. Association of PD-1, PD-1 ligands, and other features of the tumor microenvironment with response to anti-PD-1 therapy. Clin Cancer Res. 2014; 20(19):5064-5074.

29. Sun X, Sun J, Yuan W, et al. Immune cell infiltration and the expression of PD-1 and PD-L1 in primary PDGFRA-mutant gastrointestinal stromal tumors. J Gastrointest Surg. 2020. https://doi.org/10.1007/s11605-020-04860-8

30. Zhao R, Song Y, Wang Y, et al. PD-1/PD-L1 blockade rescue exhausted CD8+ T cells in gastrointestinal stromal tumours via the PI3K/Akt/mTOR signalling pathway. Cell Prolif. 2019; 52:e12571.

31. Bertucci F, Finetti P, Mamessier E, et al. PDL1 expression is an independent prognostic factor in localized GIST. Oncoimmunology. 2015; 4:e1002729.

32. Gooden MJ, de Bock GH, Leffers N, Daemen T, Nijman HW. The prognostic influence of tumour-infiltrating lymphocytes in cancer: a systematic review with meta-analysis. Br J Cancer. 2011; 105(1): 93-103.

33. Gheorghe G, Bacalbasa N, Ceobanu G, et al. Gastrointestinal stromal tumors - a mini review. Journal of Personalized Medicine. 2021;11,694. 